\title{
MODELING PLANT DEVELOPMENT WITH GENE REGULATION NETWORKS INCLUDING SIGNALING AND CELL DIVISION
}

\author{
${ }^{* 1,2}$ Mjolsness E., ${ }^{1,3}$ Jönsson H., ${ }^{2}$ Shapiro B. E., ${ }^{1}$ Meyerowitz E. M. \\ ${ }^{1}$ Division of Biology, California Institute of Technology, Pasadena, CA 91125 \\ ${ }^{2}$ Jet Propulsion Laboratory, California Institute of Technology, Pasadena, CA 91109 \\ ${ }^{3}$ Department of Theoretical Physics, Lund University, Lund, Sweden \\ E-mail: emj@caltech.edu \\ *Corresponding author
}

Keywords: Arabidopsis, Shoot apical meristem (SAM), Cellerator, computer modeling.

\section{Resume}

Motivation: The use of computer models for the understanding of biomolecular systems is increasingly important. Multicellular models are especially useful in the context of developmental biological systems.

Results: We show how a simple model of gene regulatory networks combined with models of signaling and cell division can be used to simulate behavior of a real biological system, the shoot apical meristem in Arabidopsis thaliana.

Availability: http://www-aig.jpl.nasa.gov/public/mls/cellerator

\section{Introduction}

The shoot apical meristem of Arabidopsis thaliana is an example of a developmental system which can be modeled at genetic and mechanical levels provided that suitable mathematical and computational tools are available to represent intercellular signaling, cell cycling, mechanical stresses, and a changing topology of neighborhood relationships between compartments.

In previous work we have introduced a mathematical framework for gene regulation networks combined with cell signaling [Marnellos and Mjolsness 98], and the "Cellerator" package for automatic model generation from reactions relationships [Shapiro et al 2000] and regulatory relationships along with cell division [Shapiro and Mjolsness 2001]. These tools may be combined to produce models capable simultaneously of transcriptional regulation, intercellular signaling, cell division, and mechanical deformation as appropriate to a developmental model. Here we apply this approach to developmental modeling to the case of the Arabidopsis shoot apical meristem (SAM).

\section{Model}

Generalizing from [Marnellos and Mjolsness 1998] we use the combined gene regulation and cell-cell signaling dynamics:

$$
\frac{d}{d t} v_{a}(t)=\frac{1}{\tau_{a}}\left[g\left(u_{a}+h_{a}\right)-\lambda_{a} v_{a}\right]
$$

where 


$$
u_{a}(t)=\sum_{b} T_{a b} v_{b}(t)+\sum_{I \in N b r s} \Lambda^{I} \sum_{b} \hat{T}_{a b} v_{b}^{I}(t)+\sum_{I \in N b r s} \Lambda^{I} \sum_{b} \sum_{c} \widetilde{T}_{a c}^{(1)} \widetilde{T}_{c b}^{(2)} v_{c}(t) v_{b}^{I}(t)
$$

Here $T$ is an intracellular gene regulation network, $\hat{T}$ is an intercellular network, and $\tilde{T}^{(1)}$ and $\tilde{T}^{(2)}$ represent a more detailed intercellular signaling network which separates the connection of receptors and ligands $\left(\tilde{T}^{(2)}\right)$ from the connection of receptors and nuclear pathway target genes $\left(\tilde{T}^{(1)}\right)$. To this is added a simple model for cell growth and cell division, which can be chosen from a variety of published models. The resulting system can now be simulated within Cellerator as otherwise described in [Shapiro and Mjolsness 2001]. Figure 1 shows a regular initial condition for a two-dimensional meristem simulation, including five cell types for expression domains and outer cell layers.

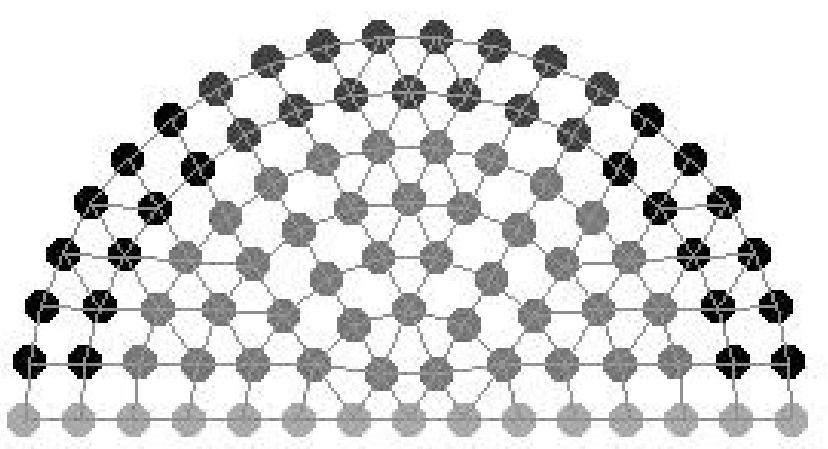

Fig 1. Meristem initial conditions. Cell type is indicated by node shading.

\section{Results and discussion}

The dynamical behavior of a much simplified 2D model, containing only central zone (light gray) and rib meristem (dark gray) starting from a rectangular grid initial condition, is shown in Figure 2.

(a)

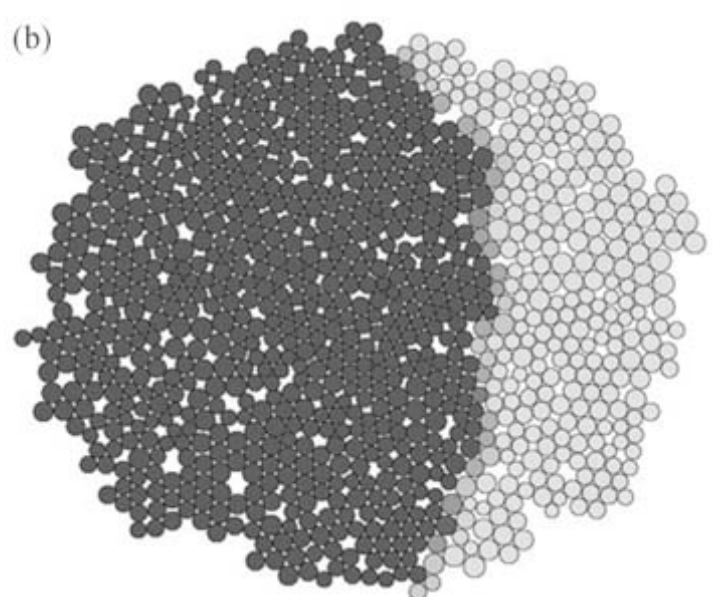

Fig. 2. Result of cell division, intercellular signaling, and intracellular gene regulation network dynamics. (a) Rectangular grid initial condition with equal numbers of cells in two expression domains. (b) Result of long-term dynamics.

In figure 2, the spatial and growth dynamics is modeled by a reversibly breakable "spring" potential between neighboring cells as described in [Shapiro and Mjolsness 2001]. Each cell also have two proteins (PA ,PB) and the protein concentrations follow the dynamics described in equation (1). Two cell types are defined by whether the 
concentration of PA is high or low indicated by colors (gray scale) in the figure. Where $\mathrm{PA}$ is high, $\mathrm{PB}$ is low and vice versa (not shown).

The cells are initiated on a two dimensional grid with a small random deviation in size and growth rate (which determines the period of the cell cycle). There are two different initial protein concentrations of the cells, dividing them into two regions of cell types (PA concentration high/low).

In figure $2 \mathrm{a}$, the first cell division has just occurred and the cells are all in nearly the state in which they were initiated. Cells have just started to grow and move from their original positions. As time elapses further, the cells start to divide and by the time of figure $2 \mathrm{~b}$ the number of cells has greatly increased. Also the intercellular interaction leads to change in protein concentrations, converting cells of one type into the other (note medium gray cells in figure $2 b$ ) when the two cell types are adjacent, as may be the case for the central zone and rib meristem of the SAM. This is also seen in figure $2 b$ by the asymmetry of the different cell type regions although they have the same division behavior. Note also the emergent hexagonal close-packing regions resulting from the nonlinear-spring elastic model described in [Shapiro and Mjolsness 2001].

Future simulation work likely to be accessible with this model include the study of different rates of cell division in central zone and rib meristem, the migration of cells from rib meristem to stem, the dynamical stability of these three domains, the incorporation of the peripheral zone, and the lineage relationships within the separate layers of the shoot apical meristem.

\section{Acknowledgments}

The research described in this paper was carried out, in part, by the Jet Propulsion Laboratory, California Institute of Technology, under contract with the U.S. National Aeronautics and Space Administration. Further support came from the Whittier Foundation, the ERATO Kitano Symbiotic Systems project, and the California Institute of Technology President's Fund. HJ was in part supported by the Knut and Alice Wallenberg Foundation through the Swegene consortium.

\section{References}

Marnellos, G. and Mjolsness, E. (1998) A Gene Network Approach to Modeling Early Neurogenesis in Drosophila. In Altman, R.B., Dunker, A.K., Hunter, L. and Klein, T. (eds), Pacific Symposium on Biocomputing, World Scientific.

Shapiro, B., Levchenko, A. and Mjolsness, E. (2001) Automatic Model Generation for Signal Transduction with Applications to MAP-Kinase Pathways. In Kitano, H. (ed), Foundations of Systems Biology, MIT Press, Cambridge, Massachusetts.

Shapiro, B. and Mjolsness, E. (2001) Developmental Simulations with Cellerator, Second International Conference on Systems Biology (ICSB). 